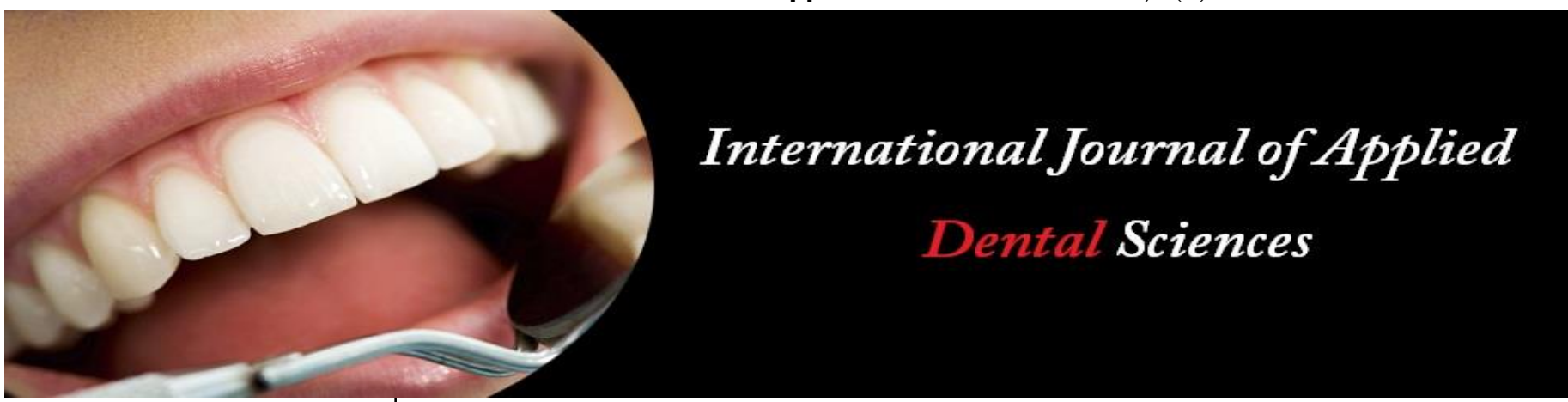

ISSN Print: 2394-7489

ISSN Online: 2394-7497

IJADS 2021; 7(4): 174-176

(C) 2021 IJADS

www.oraljournal.com

Received: 19-08-2021

Accepted: 21-09-2021

Minha Majeed Kak

Post Graduate Students,

Kothiwal Dental College and

Research Centre Moradabad,

Uttar Pradesh, India

Arya Jyoti

Post Graduate Students,

Kothiwal Dental College and

Research Centre, Moradabad,

Uttar Pradesh, India

Anurag Nath

Post Graduate Students,

Kothiwal Dental College and

Research Centre, Moradabad,

Uttar Pradesh, India

Priyanka Rastogi

Professor and Head of the

Department, Kothiwal Dental

College and Research Centre,

Moradabad, Uttar Pradesh,

India

Sachin Kumar

Professor, Kothiwal Dental College and Research Centre, Moradabad, Uttar Pradesh, India

Corresponding Author: Minha Majeed Kak Post Graduate Students, Kothiwal Dental College and Research centre Moradabad, Uttar Pradesh, India

\section{Seroprevalence of hepatitis $b, c$ and HIV among patients attending a multispecialty hospital: An 8 year old retrospective study}

\author{
Minha Majeed Kak, Arya Jyoti, Anurag Nath, Priyanka Rastogi and \\ Sachin Kumar
}

DOI: https://doi.org/10.22271/oral.2021.v7.i4c.1368

\section{Abstract}

Background: Hepatitis B, Hepatitis C and HIV infection are serious global health problems. Many risk behaviors as well as the routes of transmission for HBV and HCV infections are identical to those for HIV and other sexually transmitted diseases. They are considered as occupational risk for dental professionals and other health care workers of getting HIV, HBV or HCV infection from an infected patient and the potential transmission to susceptible patients from infected dental personnel. Due to lack of knowledge regarding the prevalence of these viral diseases among the local populations, this study was aimed to determine the prevalence of $\mathrm{HBV}, \mathrm{HCV}$ and HIV by detecting the presence of $\mathrm{HBV}$ antigen (HBsAg) and HCV antibodies (HCV-Ab) and HIV antibodies (HIV-Ab) and the relation of their presence with other factors such as gender and age among dental patients visiting a tertiary care teaching hospital in Moradabad city in Uttar Pradesh.

Materials and Methods: Case records of a total of 3000 patients were included whose serum was screened using Rapid card diagnostic kits using standard recommended procedure. HIV TRI-DOT for HIV antibodies, HEPACARD for HBsAg and HCV TRI-DOT for HCV antibodies. All rapid test positive samples were further tested by a third-generation Enzyme linked immune sorbent assay (ELISA). Data was analyzed and put for statistical analysis.

Results: The overall seroprevalence of the HIV, HBV and HCV increased over the eight year period by approximately $5 \%$. A statistical significant difference in prevalence of HIV, HCV and HBV in male and females was obtained. A high seropositivity was prevalent in the age group of 30-40 years.

Conclusion: The sero frequency of HIV, hepatitis B and C was high among patients especially from rural population visiting the tertiary health care hospital that usually go unnoticed leading to cross infection. Thus proper history taking, diagnostic tests and sterilization protocols can minimize and prevent these dreadful blood borne infections from spreading.

Keywords: seroprevalence, HIV, HBV, HCV, ELISA, Moradabad

\section{Introduction}

Hepatitis B, Hepatitis $\mathrm{C}$ and HIV infection are serious global health problems with the prevalence of hepatitis $\mathrm{B}$ virus (HBV) as $3.5 \%$ and hepatitis $\mathrm{C}$ virus (HCV) as $1 \%$. However, this prevalence shows a marked variation between different regions in the world and between countries within the same region and between different regions within the same country. Several studies have found that receiving dental treatment was a risk factor to the high prevalence of HBV, HCV and HIV [1]. Transmission of HBV from Patient to patient and Patient to dentist has been reported in dental clinics with inadequate infection control practices ${ }^{[2-4]}$ and inappropriate history given by the patients. Unsafe injections in healthcare settings, particularly in developing countries, were found to be a significant factor in the transmission of HBV, HCV and human immunodeficiency virus (HIV) ${ }^{[5,6]}$.

Hepatitis commonly causes swelling and inflammation of the liver with its chronic form leading to cirrhosis or cancer. Sometimes, people contact hepatitis with controlled or no symptoms but usually it leads to jaundice, anorexia, poor appetite and diarrhea ${ }^{[7]}$. Hepatitis can be caused by alcohol, poisons, drugs and autoimmunity but majority of cases are caused 
by viruses. Among the principal causes of severe liver disease in Hepatitis, hepatocellular carcinoma and cirrhosis-related end-stage liver disease are majorly caused by Hepatitis B virus (HBV) and hepatitis $\mathrm{C}$ virus (HCV) ${ }^{[8]}$. The HIV/AIDS epidemics are one of the largest Public Health Problem of 21 st century worldwide and vaccination for same still remains elusive. The predominant causes of morbidity and mortality among HIV patients, usually with end stage are opportunistic infections which usually do not occur in HIV-infected patients until CD4 T-cell counts have dropped to less than 200 cells/mL ${ }^{[9]}$.

\section{Materials and Methods}

This descriptive retrospective study of cases screened for serology from 2010-2018 was conducted in the Oral pathology and microbiology department of Kothiwal dental college and research hospital Moradabad. Case records of only Dental patients referred from different Departments of the Dental institution for extractions and other major and minor surgical procedures including diagnostic biopsy prior to final oral and maxillofacial surgical procedures were enrolled. Cases from the associated general hospital of this institution were excluded from the study.

Case records of a total of 3000 patients were included. The serum of the patients was screened using Rapid card diagnostic kits. For HIV antibodies, using standard recommended procedure, rapid card test as HIV TRI-DOT. HBsAg was determined by rapid card, HEPACARD (biomed industries, India). The serum was tested for HCV antibodies using standard recommended procedure, rapid card test as HCV TRI-DOT with entire test being performed in accordance with the manufacturer's instructions with adequate control. All rapid test positive samples were further tested by a third-generation Enzyme linked immune sorbent assay (ELISA). Data was analyzed using SPSS (Statistical Package of Social Science) software program version 16 and the prevalence and percentage of all variables were calculated. Chi-square test was applied to see difference by gender.

\section{Results}

Among the 3000 cases studied, 2200 (73\%) were males and $800(27 \%)$ females. (Table 1) The age ranged from 10 to 80 years with the mean age of $43.51 \pm 15.51$ years. (Table 2$)$. The prevalence of HBV was found to be $54.3 \%$ (1370 cases: $82 \%$ males and $18 \%$ females), HCV was found to be $36.6 \%$ (1100 cases: $73 \%$ males and $27 \%$ females) and HIV was found to be 9\% (270 cases: $67 \%$ males and $33 \%$ females). Table 3. An increase in the number of cases has been observed over the years from 2010-2018 (Table 4).

Table 1: Depicting the Gender distribution of cases

\begin{tabular}{|c|c|c|}
\hline Gender & Frequency & Percentage \\
\hline Male & 2200 & 73 \\
\hline Female & 800 & 27 \\
\hline
\end{tabular}

Table 2: Depicting the Age distribution of cases

\begin{tabular}{|c|c|c|}
\hline Age (years) & Frequency & Percentage \\
\hline $10-20$ & 100 & 3.3 \\
\hline $21-30$ & 700 & 23 \\
\hline $31-40$ & 1200 & 40 \\
\hline $41-50$ & 600 & 20 \\
\hline $51-60$ & 200 & 7 \\
\hline $61-70$ & 130 & 4.3 \\
\hline $71-80$ & 70 & 2.3 \\
\hline
\end{tabular}

Table 3: Depicting prevalence by Gender

\begin{tabular}{|c|c|c|c|c|c|c|}
\hline \multirow{2}{*}{ Sero positive patients } & \multicolumn{2}{|c|}{ Male } & \multicolumn{2}{|c|}{ Female } & \multirow{2}{*}{$\chi^{2}$} & \multirow{2}{*}{ p - value } \\
\hline & $\mathbf{N}$ & $\%$ & $\mathbf{N}$ & $\%$ & & \\
\hline HBV positive $(\mathrm{n}=1370)$ & 1120 & 82 & 250 & 18 & 7.890 & \multirow{3}{*}{$<0.001$} \\
\hline HCV positives $(n=1100)$ & 800 & 73 & 300 & 27 & 5.860 & \\
\hline HIV positives $(n=270)$ & 180 & 67 & 90 & 33 & 3.196 & \\
\hline
\end{tabular}

Table 4: Rise in cases over the years (2010-2018)

\begin{tabular}{|c|c|}
\hline Year & No. of cases reported \\
\hline $2010-2011$ & 130 \\
\hline $2011-2012$ & 145 \\
\hline $2012-2013$ & 210 \\
\hline $2013-2014$ & 228 \\
\hline $2014-2015$ & 343 \\
\hline $2015-2016$ & 530 \\
\hline $2016-2017$ & 564 \\
\hline $2017-2018$ & 850 \\
\hline
\end{tabular}

\section{Discussion}

Globally, there are approximately 257 million people infected with $\mathrm{HBV}$ and 71 million people infected with $\mathrm{HCV}{ }^{[1]}$ with an estimated 400 million being chronic carrier of the virus ${ }^{[7]}$. Around 1 million die due to the consequences of the infection $[10,11]$. Individual seeking dental care may be healthy or suffering from dreadful blood borne diseases like HIV, hepatitis $\mathrm{B}$ and $\mathrm{C}$ or may be carriers that cannot be identified easily either due to negligence in history taking by the doctor or not given by the patient. Such patient may act as potent sources for spreading such infection among dental health care workers and other patients in dental setups and clinics. The objective of present study was to highlight the potential hazards of HIV, HBV and HCV to the dental doctors and other associated health workers as well as to patients attending clinics.

A high sero-positivity was prevalent in the age group of 31-40 years. A fivefold increase in the number of cases has been observed over the years when clubbed together. This may be attributed to the low socioeconomic status, illiteracy and ignorance and overall poor health care ${ }^{[7]}$. $3.3 \%$ cases were found in the age range of below 20 years that may be due to negligence towards the vaccination protocols ${ }^{[1]}$. However, in one of the studies, $14.35 \%$ were below the age of 15 years and $47(6 \%)$ were below 10 years ${ }^{[12]}$.

As for as the study area is concerned, numerous unqualified medical practitioners especially dental quacks are working in peripheral areas who do not posses any knowledge about cross infection and sterilization/barrier protocols as a result of which there may be high chances of cross infection. Furthermore, each dental treatment needs to follow same sterilization protocols as any other minor/major surgical procedures which means more time, equipments, manpower and expenditure required. In some cases, history of barbers sharing and using same razors among men-folk is also documented ${ }^{[7]}$.

\section{Conclusion}

As in this retrospective study only cases referred from Dental Departments who had to undergo elective surgical procedures and were confirmed positive were included and from the results of the study it is confirmed that all the dental professionals and associated paramedical staff are constantly exposed to these blood borne viral infections and pose a threat thus all dental care professionals including doctor, paramedical staff or student need to be screened for HIV, hepatitis B and C and all sterilization protocols like use of disposable gloves, syringe etc. for all procedures must be 
followed and all instruments must be autoclaved and used as separate sets for each patients. Also, a pre-operative screening (of all patients) being prepared for minor or major surgeries for HIV, HBV and HCV should be recommended as a routine not for stigmatization, but to enable the healthcare givers make adequate preparations and take appropriate preventive measures when managing such patients and avoiding the risks of cross infection.

\section{References}

1. Amad ASH. Prevalence of hepatitis B, C and HIV among patients attending a teaching dental hospital A 7-year retrospective study from the United Arab Emirates. Saudi Med J 2018;39(5):500-5.

2. Shaw FE Jr, Barrett CL, Hamm R, Peare RB, Coleman PJ, Hadler SC et al. Lethal outbreak of hepatitis B in a dental practice. JAMA 1986;255:3260-4.

3. Redd JT, Baumbach J, Kohn W, Nainan O, Khristova M, Williams I. Patient-to-patient transmission of hepatitis B virus associated with oral surgery. $\mathbf{J}$ Infect Dis 2007;195:1311-4.

4. Radcliffe RA, Bixler D, Moorman A, Hogan VA, Greenfield VS, Gaviria DM et al. Hepatitis B virus transmissions associated with a portable dental clinic, West Virginia, J Am Dent Assoc 2009, 2013;144:1110-8.

5. Hutin YJ, Harpaz R, Drobeniuc J, Melnic A, Ray C, Favorov $\mathrm{M}$ et al. Injections given in healthcare settings as a major source of acute hepatitis B in Moldova. Int. J Epidemiol 1999;28:782-6.

6. Kane A, Lloyd J, Zaffran M, Simonsen L, Kane M. Transmission of hepatitis $\mathrm{B}$, hepatitis $\mathrm{C}$ and human immunodeficiency viruses through unsafe injections in the developing world: model-based regional estimates. Bull World Health Organ 1999;77:801-7.

7. Latoo S, Shafi M, Nazir H. Sero-prevalence of Hepatitis $\mathrm{B}$ and $\mathrm{C}$ virus among patients attending Dental clinics in Kashmir Valley. IAIM 2017;4(2):53-9.

8. Mujeeb SA, Aamir K, Mehmood K. Seroprevalence of HBV, HCV and HIV infections among college going first time voluntary blood donors. J Pak Med Assoc 2002;50(8):269-70.

9. Pratima Tripathi et al. Seroprevalence of Hepatitis B, Hepatitis $\mathrm{C}$ and HIV Infection in Tertiary Care Teaching Hospital in Moradabad (U.P). Sch J App Med Sci 2018;6(4):1405-10.

10. Lee WM. Hepatitis B virus infection. N Engl. J Med 1997;337:1733-45.

11. Mamun AIM, Fasle Akbar SM. HBeAg negative chronic Hepatitis B an overview Hepatitis B Annual 2009;6(1):131-40.

12. Rehman $\mathrm{S}$ et al. Epidemic of Hepatitis $\mathrm{C}$ in a remote village of Kashmir, India. EC Bacteriology and Virology Research 2016;2(1):54-62. 